\title{
Small Cells Handover performance in Centralized Heterogenous Network
}

\begin{abstract}
Improving the flexibility and added complexity are two terms that must be considered carefully when presenting new solution for the next generation mobile networks or (5G). Reliability, availability, latency and data rates are some of the key indicators of the performance of the new systems.

Heterogeneous network supported by computing functionality is one of the most recent added flavor topologies to LTE technology and the upcoming next generation mobile network. Mobility is yet one of the main challenges in such system.

Allowing the user to serve the world of the data without interruption while in the move between the different cells of the Heterogeneous network is required to keep high level of the user's quality of experience.
\end{abstract}

Keywords-Heterogeneous network; computing; LTE; Mobility.

\section{INTRODUCTION}

Passing through its different generations from $1 \mathrm{G}$ to $4 \mathrm{G}$, Evolution of mobile network examine different fundamental changes and challenges. System evolved from analogue network providing voice only service to an all IP packet core network providing multimedia services. During this evolution, the structure of the mobile network itself passes through different changes, in the two main parts of the structure i.e. the core network and the radio network. The way the mobile user access the network also affected by this evolution. In addition, the experience of the mobile users accessing the network also played a role in this evolution. The mobile user is the end user of the network and it is the customer of the mobile network operators (MNO), therefore, keeping a good level of experience is a fundamental requirement in order to keep a good level of revenue.

Customer satisfaction is a need for the mobile network operators (MNO), and as the mobile networks evolved, the customers become more demandable and keeping the level of satisfaction become more challenging.

Mobility is one of the most attractive attribute of the mobile network, the ability of the mobile user to move through the network and benefiting from the services is one of the charming characteristics that keeps the user attracted to the mobile networks, at the same time this attribute represent one of the most challenging areas for the mobile network operators. In order to keep the level of satisfaction, the mobile network operators must provide the mobile users with seamless connectivity and all time services, especially as the mobile devices are no longer a luxury and become an essential part of people's everyday life and business. People are using their mobile devices while on the move for different purpose and needs, they are serving the internet, checking their emails, connecting with each other's through social media, and watching videos. The integration between mobile networks and computer networks with the advanced capabilities of the devices lead to big amount of data to be generated, and the most amount of this data is due to mobile networks and the attached devices, such as mobile smart phones, tablets, wearables and many other devices. According to Cisco Visual Networking Index (VNI) 2017 [1], by 2021, that is one year after the 3GPP to submit the final specifications of the $5 \mathrm{G}$ at the ITU-R WP5D meeting in February 2020, 58\% of the population will be using the internet, 3.5 networked devices and connections per person, global IP traffic will reach 3.3 Zettabyte, traffic from wireless and mobile devices will represent $63 \%$ of total IP traffic, Smartphones will exceed 86 $\%$ that is four-fifths of mobile data traffic, and over $78 \%$ of that is three-fourths of the world's mobile data traffic will be video. Based on that and taking the requirement for the $5 \mathrm{G}$ mobile network in consideration, that include: higher connection speed of up to $10 \mathrm{Gbps}$, latency of about $1 \mathrm{~ms}$, increasing the bandwidth per unit area, $100 \%$ coverage and almost the same for availability. It can be seen that the current structure of the $4 \mathrm{G}$ represented by the structure of Lte-A will not be able to cope with such requirements, but could be the base of the future mobile network or 5G. Before the full release of $5 \mathrm{G}, 4 \mathrm{G}$ will continue to develop to support many new use cases and applications, by making some modification and enhancement on its structure, 4G can handle many new features that could be seen as $5 \mathrm{G}$ specific. Such enhancement includes the use of small cells in Heterogeneous networks, mobile edge computing and cloud computing, virtualizations and software defined networks (SDN), and network slicing.

\section{A. Heterogenous network}

Unlike the homogeneous network that consists of macrocells only, a heterogenous network is the one that composed of multiple sites types, i.e. macrocells, microcells, picocells, 
femtocells and either Wi-Fi hotspots. Such network architecture produce challenges in terms of co-channel interference and RF planning, as the various types of BTS typically sharing the same channel bandwidth [2]. One of the obstacles in the heterogenous network is the intercell interference mainly in the boundaries of the cells that can cause handover problems. Solutions such as intercell interference coordination (ICIC) and enhanced intercell interference coordination (eICIC) techniques as specified by 3 GPP specifications in release 8 and 10 respectively can be used to mitigate such issue. In ICIC techniques the signal to noise ratio (SNR) is improved at the cost of reducing the total number of resource blocks i.e. the bandwidth available for transmission, by avoiding the use of the resource blocks used by neighbouring eNodeBs. eICIC also improves the SNR by benefiting from almost blank subframe (ABS), which allow neighbouring eNodeBs to reduce their transmission during certain time intervals.

Many reasons that make a heterogenous network beneficial, such as: the difficulty of site acquisition for macrocells, site acquisition for smaller cells are easier. Increase network capacity with greater density deployments of the cells. Provide coverage at location outside of the reach of the main network or for locations of poor coverage, such as indoor location. In addition small cells can be used to provide dedicated services in the term of network slicing.

\section{B. Small cells}

A promising solution that can cope with mobile traffic explosion is the use of the small cells. As in [3], a node whose transmit power that is lower than the BS classes of macro node can be considered as small cell. In such situation Pico and Femto cells that have transmit power of $0.25 \mathrm{~W}$ and $0.1 \mathrm{~W}$ respectively are both considered as small cells, which is not the case for Microcells. 3GPP doesn't specify a separate power class for Microcell. A wide area BTS, i.e. Macrocell with reduced transmit power could be designed and considered as Microcell.

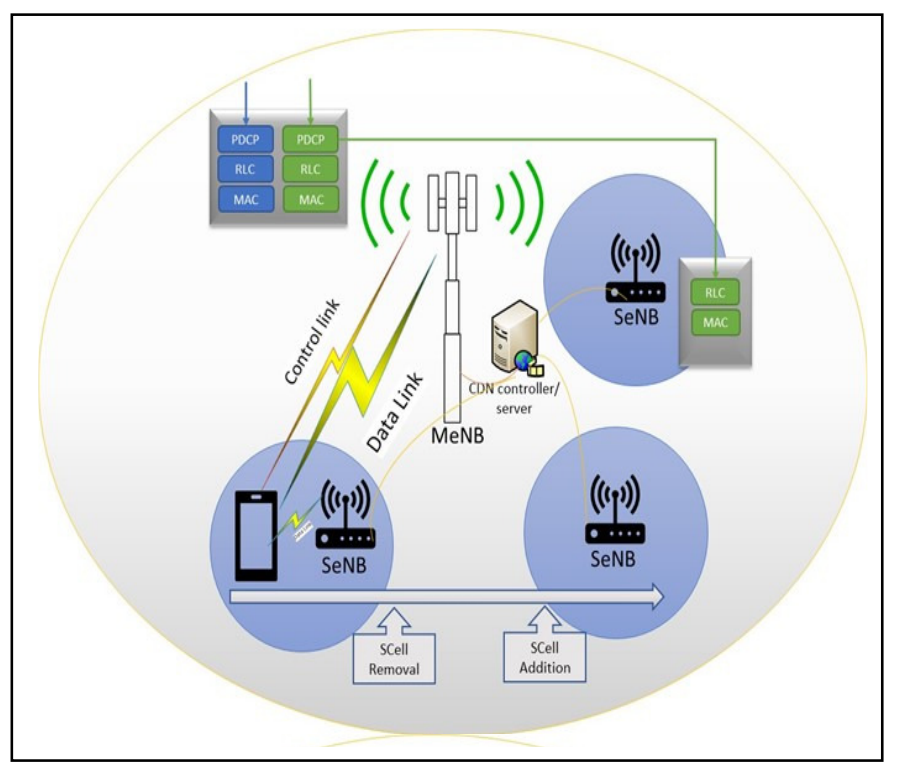

Figure 1: System Architecture
In some other scenarios, Wi-Fi hotspots [4], and remote Radio heads (RRHs) within a centralized Radio Access Network (C-RAN) are also considered for small cells deployment options.

As in [5], the scenarios target the deployment of small cells with and without the coverage of Macroells, indoor and outdoor hotspots, with both ideal and non-ideal backhaul, with the possibility of small cells to use the same or different frequency band when under the Macro cell layer. The distribution technique is also an important factor, therefore the spars and dense distribution is also considered within the 3GPP TR. Study on the impact of each architecture option will lead to better decision on how to deploy small cells and the service that could be delivered, taking in consideration the user's locations and dual connectivity in heterogeneous network.

\section{Dual connectivity}

Carrier aggregation (CA) is the most successful feature of the LTE-Advanced system that was introduced in 3GPP release 10. CA increase the channel bandwidth by combining multiple RF carriers coming from the same co-located eNB, by giving the UE the ability to receive and transmit multiple signals in both directions i.e. downlink and uplink [2][4]. Dual connectivity is an interesting and important extension to the CA principle that was adopted in release 12 of the 3GPP, in which the UE will have the ability to be connected with both macro and small cell simultaneously. This will allow the maximum data rate to be aggregated from the macro and small cells layers. With such feature small cells could be used in the most efficient way as the macro cell could be used to maintain the coverage keeping the UE connected all the time to the system even in the absence of the small cell layer coverage. Different architecture options for the deployment of such heterogeneous network that use small cells layer and UE dual connectivity feature as reported in 3GPP TR 36.842 [6]. Benefit of such architecture, based on different scenarios include: continuous connectivity, reduce signalling overhead with core network, increase the peak data rate, the possibility to use the small cells layer for dedicated services such as content delivery. Although such architecture brings many benefits, yet it brings many challenges as it increases the system complexity. Challenges such as: Efficient distribution of the small cells and the discovery scenario of such small cells by the UE that may exhaust the battery power, the miss measurement and exchange of the signals between the Macro and small cells layers may lead to ping pong situation and handover problems during the mobility.

\section{RELATED WORK}

Wireless and mobile networks have an increasing impact in the world, smart devices connected to such network become more powerful and are evolving in a way that surpass the evolution of the mobile network system, benefiting from the combination of the computing technology and the powerful capabilities of the software programmes. These powerful mobile devices become the primary point of access to the internet system. Wireless mobile devices can be found 
everywhere, it can be found in our homes, cars and offices and it is wearable, portable, and attached to mobile equipment and things like drones. The attractive features of such devices that make them popular and widely adopted by users is their light weight, wireless connection and mobility. While in the move, devices with such exiting features are exchanging information and generating huge amount of data, taking in consideration the new applications and services provided to the mobile users by different service providers, in addition to the use of data in different fields by different parties such as marketing, results in creating a more demandable users that requires all time connection with more bandwidth. this become a burden to the current wireless and mobile system, and therefore the design of next generation mobile network must took these issues in consideration. In order to support such demands, the next generation mobile network will be a multipurpose system with network slicing that provide dedicate services specific to the need of each customer. In order to implement such network, mobile system need to merge and benefit from the capabilities of the computer system in addition to other access network techniques. As the next generation mobile network will be a key enabler to other systems and the base for the Internet of Things (IoT) it should be designed to integrate networking, computing and storage resources into a programmable unified infrastructure that will allow for an optimized usage of resources and be able to process the huge amount of generated data by mobile users [7].

In this demand many companies, and institutes are grouped together in an alliance or working groups, in an efforts to provide their vision for the next generation mobile network. in addition to many academic work presented for the same purpose. Most of the work is based on the system architecture of the current Lte-A system and traying to solve the problem of the data delivery especially when the user is moving. A document presented by Ericsson to the 3GPP RAN, suggest a technique for CP and UP separation between MeNB and SeNB to increase mobility robustness and minimize UE context transfer [8]. At the same event FiberHome Technologies Group, presents a study for Small cell discovery in HetNet based on existed uplink signal, based on cell listening to reduce Signalling overhead [9]. In [10] the authors presented a work for small cells dual connectivity with bearer split in the uplink direction in order to increase the user throughput by means of coordination between the cells.

In the same scenario authors in [11] proposed a flow control algorithm to forward the data from the MeNB to the SeNB, results shows that there is a trade-off between user throughput and latency. The authors in [12] presented a scenario for dual connectivity in which the processing of the data and the control of the SC is within a controller on the edge of the network i.e. mobile edge computing (MEC), The results show that the proposed scheme can reduce the packet loss and latency when routing full load in the network.

In terms of computing capabilities to support next generation mobile system, separation of CP and UP techniques supported by software defined network (SDN) to enable content caching was presented in [13] The separation of CP from UP will eliminate the need for mobile dedicated solutions as it removes the mobile tunnelling to allow dynamic relocation of the cache.

In the road to $5 \mathrm{G}$, a cache-enabled wireless heterogeneous network (HetNet) with (SDN) and split of the CP and UP is proposed in [14], where the macro cell and SCs with different cache abilities are overlaid and cooperated together in the backhaul. Based on the results, it was found that the proposed network has much higher throughput and energy efficiency than current LTE networks. Fundamental trade-offs exist between the throughput and the density of SCs.

\section{ARCHITECTURE}

The architecture is based on the 3GPP LTE-A Evolved Packet System (EPS), with the same main components for radio and core networks, (the core network deployment in OPNET consist of only one node called the EPC). In the radio network, and in a similar way to scenario\#2 in the 3GPP TR 36.842 V12.0.0 (2013-12) (Release 12)[6], small cells will be distributed as hot spot covering a specific areas under the coverage of the macro-cell layer, and using different frequency of the macro cell. The small cell layer with frequency (F2) will be located at the centre of the hot spot, where the macro with frequency (F1) will be like an umbrella covering the small cells layer. In our assumption the macro and small cells layers are connected via an ideal backhaul.

In this scenario, a UE with dual connectivity will maintain an RRC CONNECTION with the MeNB at all times, while receiving user plane data from the MeNB and SeNBs. Hence there will be only one S1-MME connection per UE. In such way, the mobility of the UE will be controlled through the $\mathrm{MeNB}$, as the MeNB will be responsible of the RRC signalling with the MME and there is no need to move the RRC context of the UE between the SeNB's. in this manner, handover between SeNB's will be like adding and removing new cells as all the information and resource management (RRM) are in the MeNB server.

The main service required by the UE will be the responsibility of the MeNB as it keeps all time connection with the UE, while SeNBs could be used for specific services such as content delivery.

In this scenario there will be a C-Plane and U-plane separation, and there will be a barrier split as well. As it is the

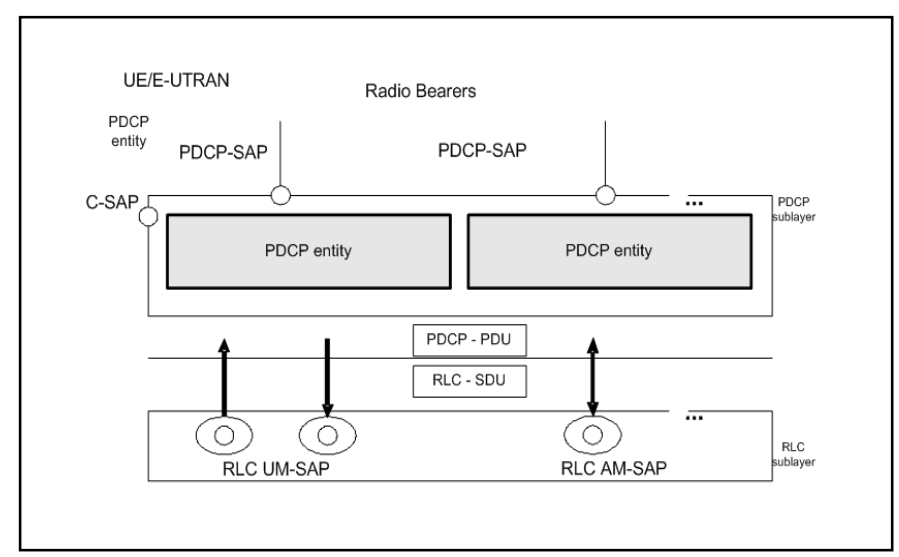

Figure 2: PDCP Layer, Structure view [15] 
responsibility of the control (C-plane) to transfer signalling messages using the RRC layer of the control plane protocol stack, by the means of different Signalling Radio bearers (SRB) between the UE and MeNB, examples of such RRC signalling include, paging, RRC connection establishment, reconfiguration and release. The Packet Data Convergence Protocol (PDCP) layer (Fig. 2) within its responsibility is to transfer the user plane and control plane data by the mean of PDCP entities, several PDCP entities may be defined for each UE, and each PDCP entity is carrying data for one data bearer In this manner we could get a centralized radio access network similar to the C-RAN with RRH, but instead of the RRH that just have a RF layer, the SeNBs will have a protocol stack that eliminate the need for the RRC and PDCP layers and have the RLC, MAC and PHY layers. The Service Data Units (SDUs) coming from the PDCP layer are then encapsulated and directed to the RLC layers in each eNB that will convert them to Protocol Data Units (PDUs). Passing through the rest of the protocol stack. UE will receive the data from one or more eNB, assuming the scheduler in the MeNB server has shared the total set of the resource blocks, separate IFFT functionality could be used to separate between the signals.

In terms of mobility, a UE in RRC connected mode relies on network controlled UE assisted mobility, in which the network informs the UE via a dedicated RRC signals when it has to perform handover process to the required cell. When a UE follows a certain trajectory while in dual connectivity, it can have its PCell on the macro layer, i.e. MeNB, while SCell can be configured within the small cell layer, i.e. SeNB. Two types of handover could occur within such architecture, between MeNBs and between SeNBs. The MeNB handover is based on the RSRP A3, while addition and removal of SeNBs are based on RSRQ A4 \& A2, respectively. Network will keep control of the mobility of the UE as the MeNB is controlling the C-plane. Consider adding the computation and storage capability, from other working groups, e.g. ETSI MEC, a CDN network controller could be located within the macro cell layer (MeNB). Information about the network and its users will be gathered at the controller. Such information includes mobility, number of users per cell, list of candidate cells for under the MeNB coverage. This can provide significant impact in the UE data rate. When those SeNB are well known by the control server of the MeNB and sharing the same C-plane, all the information necessary to provide the required service can be started directly after the UE is under the SeNB coverage and the triggering criteria for SCell addition is configured by the MeNB controller by mean of certain event e.g. A4. In this case RRM measurement are not reported to the core network.

Similar architecture for controlled RRM and handover between small cells could be a promising method reduce signalling overhead towards the core network, as well for increasing the data rate per UE

\section{PERFORMANCE EVALUATION}

After introducing some basic terminologies and concepts, a study of the UE behaviour moving under the coverage of
MeNB and receiving data from a content server attached to that MeNB while experiencing hand over procedure between the small cells. The study is based on the system architecture explained in section III of this work and will be implemented using Riverbed Modeler formally well known as OPNET Modeler.

\section{A. Simulation Setup}

The Riverbed LTE Modeler 18.7 is based on the 3GPP Rel.8. therefore, the system has been modified to support dual connectivity using the device creator operation to create a custom LTE nodes for our network [ OPNET documentation create Custom Device Model Operation].

An LTE system simulation scenario that consist of 1 MeNB that will represent the coverage area where the UE will move, and 4 SeNBs that will represent the hotspot dedicated for specific service and randomly distributed under the MeNB coverage using frequency band different from the one used by the MeNB.

The UE will keep RRC-Connection with MeNB at all time of the simulation, thus having $1 \mathrm{~S} 1-\mathrm{C}$ interface with the MME through the MeNB. Mobility management function tracks the location and activity of a UE in the network. The core network needs to know the location of a UE for traffic transmission. A measurement of RSRP and RSRQ are supported, thus the UE continuously measures RSRP and RSRQ for all cells within the range. The MeNB is configured to receive periodic measurement from the UE. Handover is initiated and controlled by the MeNB, with assistance from the UE. Data forwarding while handover is supported. The report_interval function and num_reports attributes within the Riverbed modeler govern the number of reports sent to the MeNB. UE will perform cell search and generate reports, when the reported measurements violate the handover triggers, the MeNB decides to handover the UE to a different cell from a list of the SeNB in its attached server.

The system performance of a UE under the coverage of MeNB and receiving data from both MeNB and one of the SeNBs while moving in a specified defined trajectory that insure the UE will be under the coverage of one SeNB within the MeNB coverage area.

Table I: Simulation parameters

\begin{tabular}{|l|l|}
\hline \multicolumn{1}{|c|}{ Parameters } & \multicolumn{1}{c|}{ Values } \\
\hline Scenario & $\begin{array}{l}\text { scenario\#2 in the 3GPP TR 36.842 V12.0.0 } \\
(2013-12)\end{array}$ \\
\hline Deployment & $\begin{array}{l}13 \text {-sector MeNB site } \\
4 \text { SeNB sites per macro cell area }\end{array}$ \\
\hline $\begin{array}{l}\text { MeNB Carrier Frequency } \\
\text { (F1) }\end{array}$ & $2 \mathrm{GHz}$ \\
\hline $\begin{array}{l}\text { SeNB Carrier Frequency } \\
\text { (F2) }\end{array}$ & $3.5 \mathrm{GHz}$ \\
\hline LTE B.W./Duplexing & $20 \mathrm{MHz} / \mathrm{FDD}$ \\
\hline MeNB Tx Power & $46 \mathrm{dBm}$ \\
\hline SeNB Tx Power & $30 \mathrm{dBm}$ \\
\hline UE Tx Power & $23 \mathrm{dBm}$ \\
\hline Traffic model & File download traffic over TCP \\
\hline File Inter-arrival Time & Exponential \\
\hline Service type & As requested by UE \\
\hline
\end{tabular}




\section{B. Result discussion and analysis}

In here; the scheme proposed is analysed based on the discussed settings and scenarios explained in the proceeded sections of this paper. We also examine IP data packets (both sent and received) all over the LTE network, considering both FTP load and video contents.

The key performance factors chosen here are the throughput and accumulative network burden. The first scenario proposed a low load traffic network configuration presented by FTP. This is to decrease the probability of packet loss due to either the buffer over-flow or, when there is traffic congestion, repeated transmissions.

Fig 3 below shows the response of the network in terms of traffic for the scenarios. it also illustrates the associated MeNB and handovers between SeNBs .It can be observed that the load in packets/sec and the response of the network is acceptable when the UE is connected to the M-eNodeB provided that no association with any SeNB in the network is set, it jumps higher when the UE's start receiving data from SeNB. This is the expected response as the burden decrease on the M-eNodeB assuming that same FTP file is routed in the network in every scenario. Whilst the incremental of SeNBs number in the entire network significantly drops the delay as the time elapsed to fetch data from the cloud is narrowed or sub-zeroed keeping in mind that the handover processing period is too short and could be neglected.

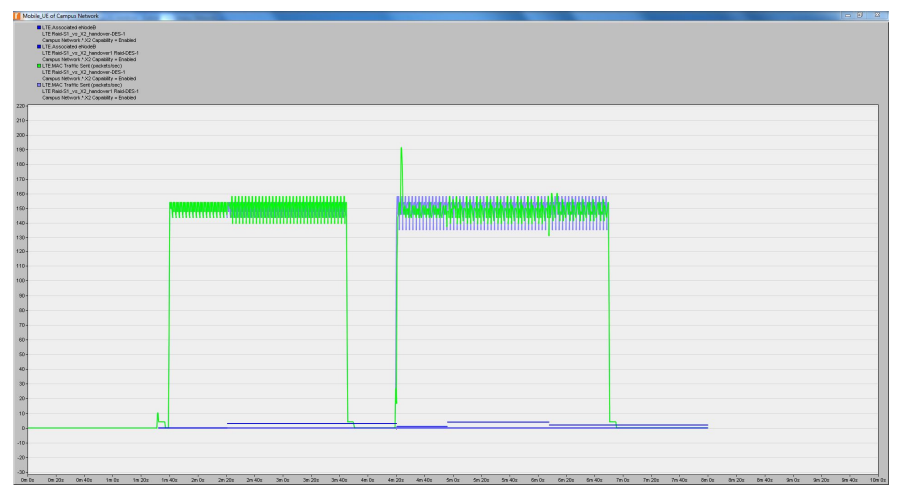

Fig 3 Throughput and associated eNB

In the next scenario a full load is deployed in the network, with the same network parameters and conditions, the network showed better cooperation and explicit maximum efficiency.

Fig.4 below illustrates the throughput delivered in bits/seconds. It can be observed that the throughput is increasing when the UE associates with SeNB

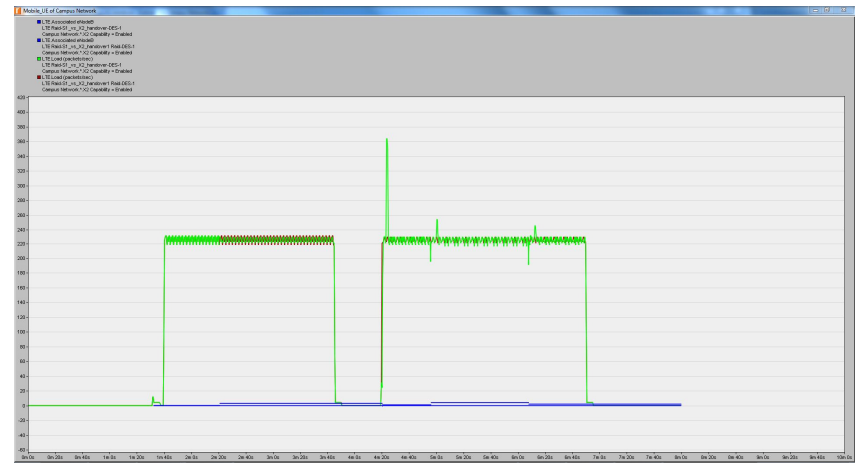

Fig 4 Throughput and associated eNB

Logically, the throughput (bits/sec) increases in 2 cases

- When the data traffic increases.

- When the elapsed time decreases.

Hence in our model, when the dual connectivity is triggered in the MeNB the network delivers and performs relatively slow at the begging of the simulation. However, it starts to perform even better in the second scenario when the DL is connected to the SeNB after $15 \%$ of the simulation time, this is due to SeNB initialization and time spent fetching the content from the cloud.

Finally we experienced the case when the same network runs and routes non live video content in a full load scheme in its data plane. Fig. 5 below shows the response of the network in terms of E2E delay for the scenarios. It can be noted that when the UE is connected to the MeNB alone, and as long as its out of the coverage of any SeNB; the delay is very high. However, the delay is acceptable when one SeNB is connected. On the other hand the delay has dropped remarkably when using the proposed model and SeNB are distributed in the network.

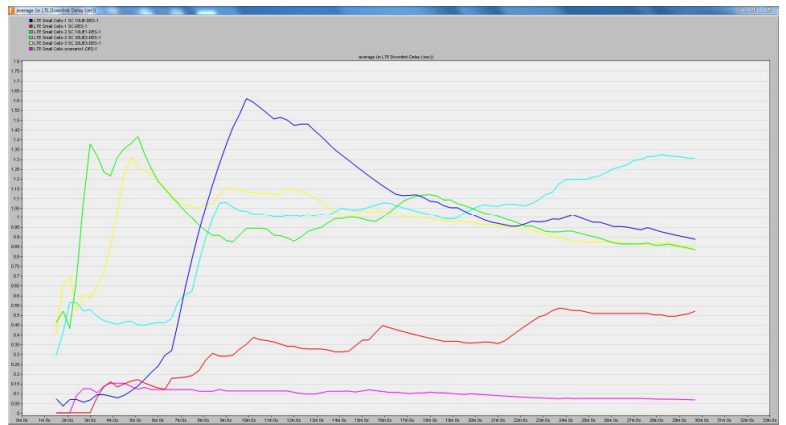

Fig 5 E2E delay

IV Conclusion

The convergence between the IT and Telecommunication Technologies bring new capabilities that will enable the deployment of new services at the edge of the network. In this paper we introduced a new transmission/reception scheme 
revolving around the CoMP concepts when UL and DL links are physically separated into MeNB and SeNB in which the processing of the data and the control of the $\mathrm{SC}$ is within a controller at the edge of the network. When comparing the data traffic received in the DL direction under many network circumstances, it can be noted that the proposed method has successfully enhanced the performance of the network and suppressed any performance degrading that may occur due to a sudden high network load or conditions. The outcomes demonstrate that the scheme put forward in this paper can decrease the packet loss the latency. When routing full load in the network, and the model becomes more efficient and delivers better results when the number of users is increased.

\section{References}

[1] Cisco Visual Networking Index: Global Mobile Data Traffic Forecast Update, 2016-2021

[2] Chris Johnson, "Long Term Evolution In Bullets", 2nd ed., ver. 1. Northampton: England, 2012.

[3] 3GPP TR 36.932 V13.0.0 (2015-12)

[4] Harri Holma, et. Al., "LTE Small Cell Optimization : 3GPP Evolution to Release 13," John Wiley \& Sons, 2015.
[5] 3GPP TR 36.932 V14.0.0 (2017-03)

[6] 3GPP TR 36.842 V12.0.0 (2013-12)

[7] Raed Sahib Sadoon, "Explosion of Data (BIGDATA)", Chapter "3" in the "Internet of Things and Big Data Analysis: Recent Trends and Challenges", Nov, 2016, ISBN-10:0692809929.

[8] Ericsson, Tdoc R2-123706, 3GPP TSG-RAN WG2 \# 83

[9] FiberHome Technologies Group, Small cell discovery in HetNet based on existed uplink signal, 3GPP TSG-RAN WG2 \#83 R2-132295, Barcelona, Spain, 19th - 23th August 2013

[10] S. C. Jha, K. Sivanesan, R. Vannithamby and A. T. Koc, "Dual Connectivity in LTE small cell networks," 2014 IEEE Globecom Workshops (GC Wkshps), Austin, TX, 2014, pp. 1205-1210. doi: 10.1109/GLOCOMW.2014.7063597

[11] H. Wang, C. Rosa, and K. I. Pedersen, "Dual connectivity for LTEadvanced heterogeneous networks," Wireless Networks, vol. 22, no. 4, pp. 1315-1328, Aug. 2015.

[12] R. Saadoon, R. Sakat and M. Abbod, "Small cell deployment for data only transmission assisted by mobile edge computing functionality," 2017 Sixth International Conference on Future Generation Communication Technologies (FGCT), Dublin, 2017, pp. 1-6.

[13] J. Costa-Requena, M. Kimmerlin, J. Manner and R. Kantola, "SDN optimized caching in LTE mobile networks," 2014 International Conference on Information and Communication Technology Convergence (ICTC), Busan, 2014, pp. 128-132.

[14] J. Zhang, X. Zhang and W. Wang, "Cache-Enabled Software Defined Heterogeneous Networks for Green and Flexible 5G Networks," in IEEE $\begin{array}{llll}\text { Access, vol. 4, pp. 3591-3604, } 2016 . & \end{array}$ doi: 10.1109/ACCESS.2016.2588883

[15] 3GPP TS 36.323 V8.6.0 Rel. 8 